\title{
ANÁLISIS TECNO-MORFOLÓGICO Y DE MATERIAS PRIMAS DE LOS ARTEFACTOS LÍTICOS DEL SITIO LAGUNA DE LA FLECHA 10 (LAGO MUSTERS, CHUBUT)
}

\author{
MARIANO R. REYES" \& SANTIAGO PERALTA G.
}

\section{RESUMEN}

Este trabajo se encuadra dentro de un proyecto general orientado al análisis del aprovechamiento de los ambientes fluviales por parte de los grupos cazadores-recolectores en el Holoceno tardío. El área de trabajo elegida es la cuenca del lago Musters (Chubut, Argentina) y el objetivo central es verificar la existencia de diversificación económica y conocer la forma en que fue utilizado este particular ambiente.

Los resultados que aquí se presentan se refieren a estudios del material lítico del sitio Laguna de La Flecha 10 (LF10), ubicado en el sector noreste del lago y asociado a lentes de fogón. El carbón recuperado en una de ellas, perteneciente al componente inferior, fue datado en ca. 1.140 años AP.

En una primera aproximación para conocer la organización de la tecnología lítica de estas ocupaciones, a través del análisis tecno-morfológico del conjunto artefactual, se caracteriza su diversidad tipológica y se estudian las materias primas utilizadas.

Los resultados de este estudio dan cuenta de una alta diversidad artefactual y de la presencia de ciertos tipos de instrumentos que permiten inferir una utilización de tipo residencial del sitio, y caracterizar a LF10 como un campamento base, en el que se realizaron múltiples actividades. Sin embargo, teniendo en cuenta el reducido tamaño del conjunto artefactual y, especialmente, el bajo número de instrumentos, éste habría sido ocupado durante lapsos de tiempo corto. Por otro lado, el uso de materias primas locales haría pensar en un tipo de aprovisionamiento del material lítico no especializado, habiéndose realizado probablemente junto con otras actividades.

PALABRAS CLAVE: Cazadores-recolectores, tecnología lítica, tipología, lago Musters, Holoceno tardío.

\section{TECHNO-MORPHOLOGICAL ANALYSIS AND RAW MATERIALS OF LITHIC ARTIFACTS FROM LAGUNA DE LA FLECHA 10 SITE (LAGO MUSTERS, CHUBUT)}

\section{ABSTRACT}

This research is part of a general project about the utilization of fluvial environments by huntergatherers in the area of Musters Lake (Chubut, Argentina) during the late Holocene. The principal aim 
is to assess the existence of economic diversification and to establish the way in which this particular environment was used.

The results presented here refer to the studies of the lithic material of Laguna de La Flecha 10 (LF10), a site located in the north-east area of the lake. The site is associated with hearths dated ca. 1.140 years BP.

In order to characterize the organization of lithic technology of the occupations, by the way of the techno-morphologic analysis of the artefactual assemblage, we assess assemblage typological diversity and raw material composition.

The results of this study indicate a high artefactual diversity and the types of instruments represented suggest a residential utilization of the site. This would allow us to characterize LF10 as a base camp, where many activities were performed. Nevertheless, considering the small size of the artefactual set, and specially the low number of instruments, we conclude that it had been occupied for short periods of time. In addition, we consider that the local raw material used was acquired within an embedded strategy.

KEY WORDS: Hunter-gatherers, lithic technology, typology, Musters lake, Late Holocene.

\section{INTRODUCCIÓN}

Este trabajo se enmarca en un proyecto general iniciado en el año 2005, cuyo objetivo es conocer la antigüedad y características de la ocupación humana de la cuenca del lago Musters. Este se encuentra ubicado en la meseta central patagónica, más precisamente en el sector centrosur de la provincia del Chubut, a $45^{\circ} 25^{\prime}$ de latitud Sur y $69^{\circ} 11^{\prime}$ de longitud Oeste, dentro de una gran depresión de origen estructural y eólico (Gran Bajo de Sarmiento, Argentina), limitada al Oeste por las sierras de San Bernardo y al Este por la Meseta Espinosa. Junto con el lago Colhué Huapi conforma la fase terminal de la actual cuenca endorreica del río Senguer (Fig. 1).

A diferencia de la mayoría de los lagos patagónicos, que están ubicados más próximos a la Cordillera de los Andes, con condiciones climáticas más rigurosas y una conexión más directa con las aguas de deshielo, que los hacen ecológicamente más pobres (lagos oligotróficos), su ubicación dentro de la meseta central patagónica, le confiere a este lago características particulares en cuanto a su productividad, y que permiten clasificarlo como lago mesotrófico (Quirós 1988). Esta mayor productividad haría atractiva la explotación de sus recursos por parte de las poblaciones de cazadores-recolectores. En este sentido, dentro de este proyecto se plantea explorar indicadores de intensificación económica de grupos cazadoresrecolectores producto de la explotación de recursos fluviales propios del lago (peces, coipos, aves, etc.), que den cuenta de posibles fenómenos de diversificación económica a lo largo del tiempo (Flannery 1969; Smith 1985; Stiner et al. 2000). A su vez, se evalúa la interacción con otros ambientes, como la costa y la cordillera, para conocer el papel que jugó esta cuenca en el ciclo de movilidad de estos grupos sociales.

Teniendo en cuenta la ausencia de estudios sistemáticos previos en la cuenca, este trabajo constituye una de las aproximaciones iniciales para conocer la organización de la tecnología lítica (Binford 1979, 1980; Nelson 1991) de los grupos que habitaron el área. Aquí se presentan los resultados de los análisis tecno-morfológicos (Aschero 1975, 1983) y de materias primas del conjunto lítico del sitio Laguna de La Flecha 10 (LF10). A partir de estos resultados, se abordan algunos aspectos relacionados a la organización de la tecnología lítica y funcionalidad del sitio.

\section{ANTECEDENTES DEL ÁREA DE ESTUDIO}

Con respecto a los antecedentes de la investigación arqueológica en la región, los primeros datos provienen de Outes (1905), quien menciona la presencia de sitios arqueológicos en las dunas lacustres de las proximidades de los lagos Musters y Colhué Huapi. Se conocen además tres antiguas publicaciones: Vignati (1950), Bórmida (1956) y Molina (1967-70), y una breve mención de González (1953:144) en donde habla de la existencia de abundantes restos de peces asociados a fogones en proximidades del nacimiento del 


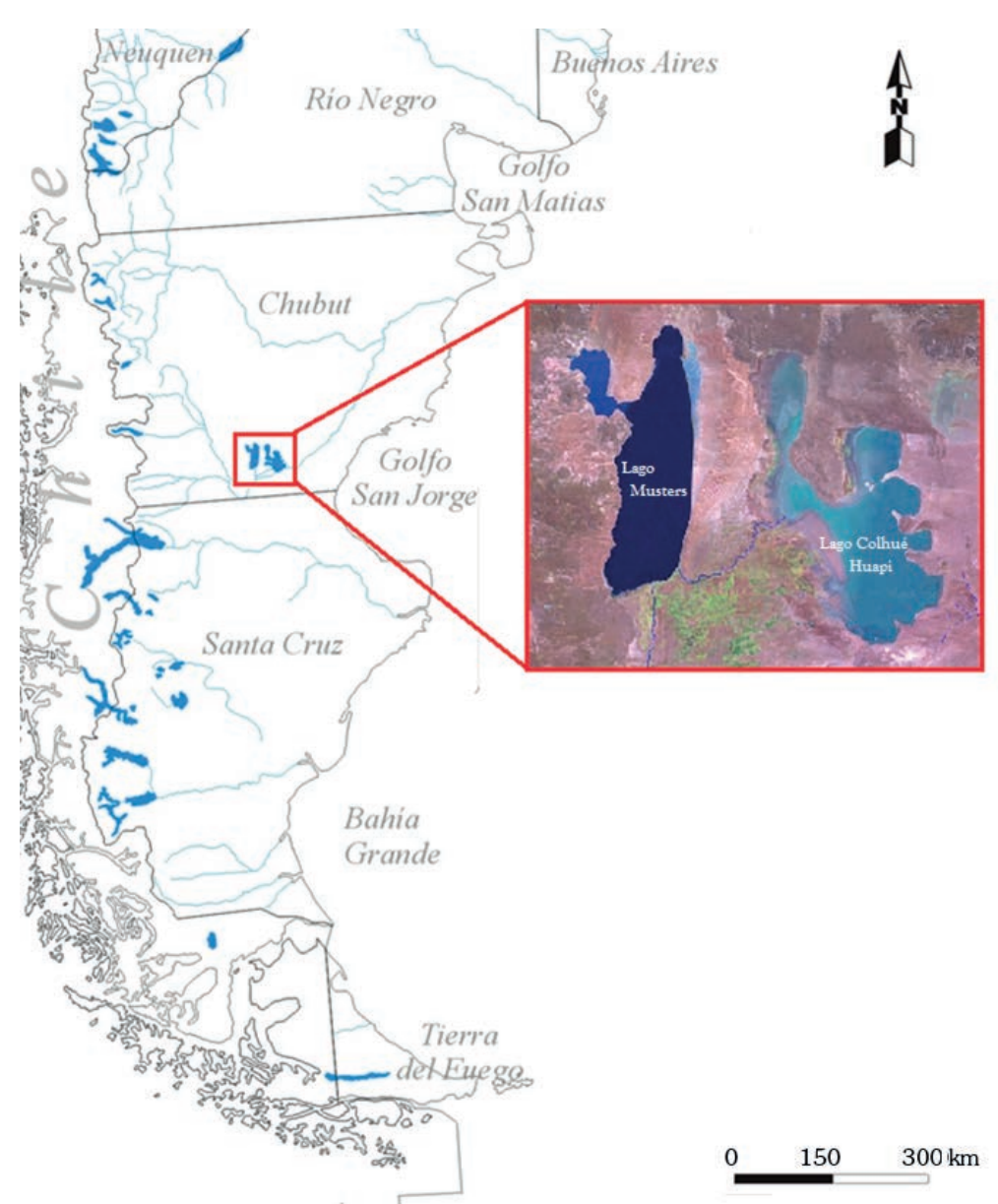

Fig. 1. Cuencas de los lagos Musters y Colhué Huapi.

río Chico, en el lago Colhué Huapi. En el caso de Vignati, este menciona la existencia, sobre la margen sur de ese mismo lago, de abundantes entierros humanos y material lítico en superficie $y$, junto al nacimiento del río Chico, de varios fragmentos de alfarería grabada. Bórmida y Molina se ocupan de describir arpones construidos en huesos de mamíferos terrestres, hallados por coleccionistas en las costas de ambos lagos. Cabe mencionar que, recientemente, han comenzado a desarrollarse investigaciones sistemáticas en el lago Colhué Huapi y el río Chico, coordinadas por Ana Margarita Aguerre y Cecilia Pérez de Micou (Castro et al. 2007; Pérez de Micou et al. 2009).

En 2005 se inician los trabajos del proyecto dirigido por el Dr. Eduardo Moreno, denominado "Búsqueda de indicadores de diversificación económica prehistórica en las cuencas de los lagos
Musters y Colhué Huapi (Chubut, Argentina)", que representa la primera investigación arqueológica sistemática en el área del lago Musters. Hasta el momento se han realizado trabajos de prospección, sondeos, excavaciones y estudios experimentales de tafonomía y procesamiento de peces (Moreno et al. 2007; Moreno \& Pérez 2010).

En general, el sector estudiado de la cuenca muestra una densa cobertura eólica, que reduce la visibilidad $y$, al mismo tiempo, protege los materiales arqueológicos. Los trabajos de prospección realizados hasta el momento han cubierto aproximadamente el $80 \%$ del perímetro del lago. Estas prospecciones han permitido detectar 132 sitios arqueológicos, tanto estratigráficos como superficiales, con abundante material, principalmente lítico, en forma de desechos de talla y artefactos formatizados como raspadores, 
puntas de proyectil, cuchillos, raederas, bolas de boleadora, molinos y manos de molino.

Si bien se hallaron sitios en todos los sectores prospectados, las mayores concentraciones se observaron en las localidades Delta del Arroyo Vulcana, Bajo del Avestruz y Laguna de La Flecha (Fig. 2). Todos los sitios están a cielo abierto, detectándose varios de ellos en estratigrafía. Hasta el momento, los trabajos se han concentrado en tres sitios, pertenecientes a las localidades mencionadas. Ellos son: Delta Vulcana 1, en el que se han realizaron excavaciones sistemáticas, y ya cuenta con un análisis de su conjunto artefactual lítico (Reyes et al. 2013); Bajo del Avestruz 4, sobre el que se realizaron varios sondeos, y cuyos materiales resta estudiar; y Laguna de La Flecha 10, al que pertenecen los materiales líticos que se analizan en este trabajo.

\section{Localidad laguna de la Flecha}

Se ubica en el sector noreste del lago Musters (Fig. 2), sobre la margen este de la Laguna de La
Flecha, un extenso cuerpo de agua somera, que se separa del lago mencionado (Moreno \& Pérez op. cit.). Se trata de un área que presenta un suelo limoarenoso, cubierto parcialmente por vegetación de tipo achaparrada, típica de la cuenca, y con una suave pendiente en dirección a la laguna. Este declive junto con las características composicionales del suelo determinaron la presencia de cárcavas con sentido este-oeste, algunas muy profundas, y que culminan en la laguna.

A diferencia de lo que ocurre en la mayor parte de la cuenca, principalmente en la margen oeste del lago, este sector sufre fuertes fenómenos erosivos. Esto, si bien permite una muy buena visibilidad, produce a su vez un fuerte impacto sobre el registro arqueológico, tanto en las relaciones espaciales entre los restos, como en la conservación de los mismos. En la localidad se han detectado 12 sitios, la mayoría de los cuales no poseerían registro en estratigrafía (Moreno et al. op. cit.), habiéndose corroborado esta expectativa, hasta el momento, solo en el sitio que aquí presentamos. En general, los sitios muestran en superficie escasos restos

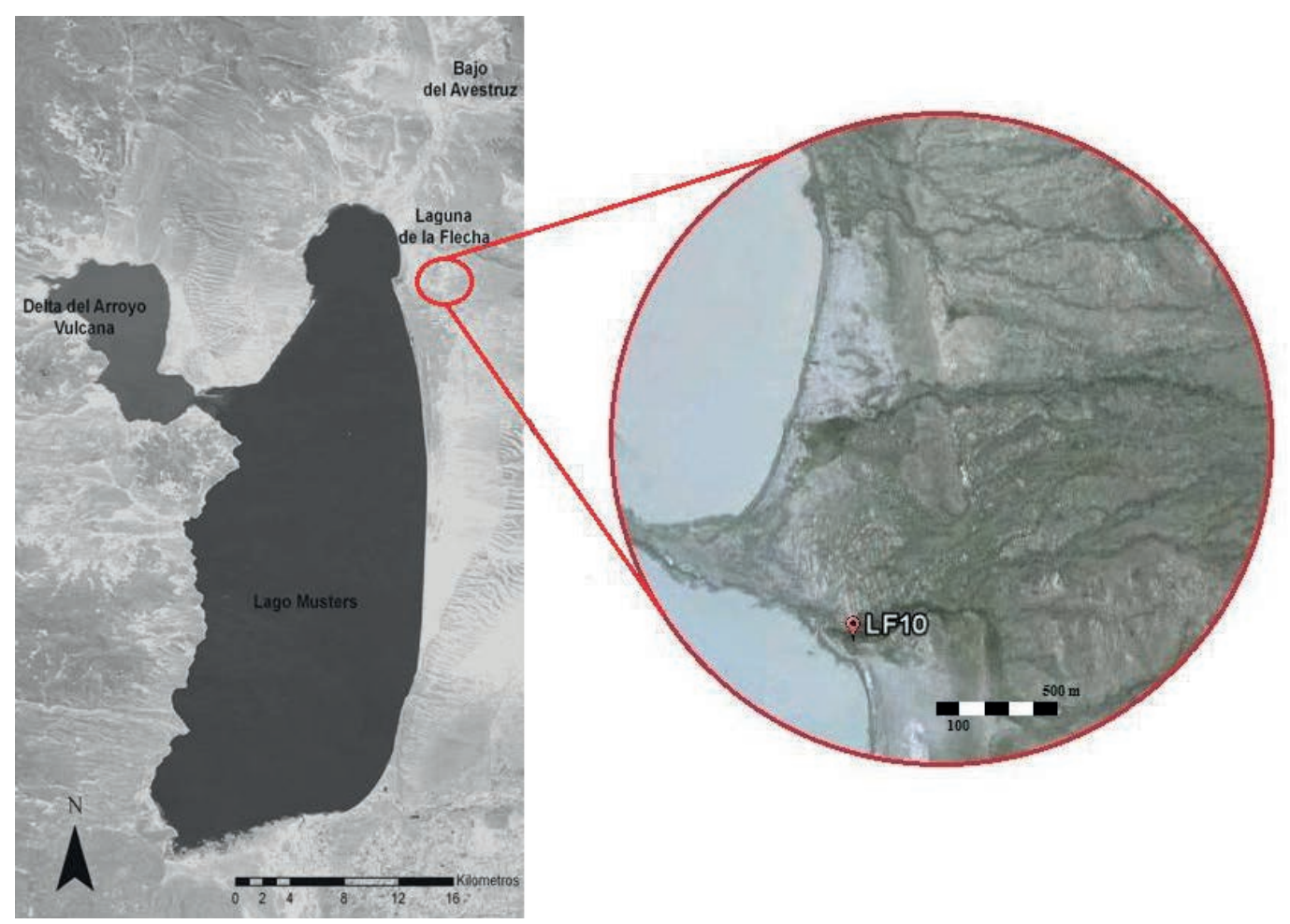

Fig. 2. Imagen satelital del lago Musters donde puede apreciarse la ubicación de las tres localidades arqueológicas mencionadas, e imagen ampliada de la Localidad Laguna de La Flecha, con la ubicación del sitio LF10. 
líticos y, en algunos casos, óseos.

Con respecto a la tecnología lítica, se hallaron diversos artefactos, en su mayoría confeccionados con distintas variedades de sílices. Los artefactos más frecuentes corresponden a desechos de talla (lascas) y diversos instrumentos, principalmente: raspadores, cuchillos, raederas, puntas de proyectil y bolas de boleadora. También fueron hallados algunos instrumentos de molienda y tiestos cerámicos, los que indicarían en principio, la existencia de ocupaciones correspondientes al Holoceno tardío. En general, los materiales se presentan formando pequeñas concentraciones en lugares erosionados, y en algunos casos asociados a lentes de fogón subsuperficiales.

\section{Sitio Laguna de la Flecha 10}

Se encuentra ubicado en el sector noreste de la laguna, a $45^{\circ} 16^{\circ} 27,59^{--}$de latitud sur y a $69^{\circ} 05^{-31}, 74^{--}$de longitud oeste, a unos $20 \mathrm{~m}$ de su costa actual, y entre esta y un antiguo cordón litoral, sobre un terreno que se eleva unos tres metros en promedio por encima del nivel de la laguna (Fig. 2). Posee una extensión aproximada de $160 \mathrm{~m}$ de longitud por $60 \mathrm{~m}$ de ancho, y está atravesado por una cárcava que alcanza en algunos sectores, aproximadamente, los $2 \mathrm{~m}$ de profundidad y $15 \mathrm{~m}$ de ancho, y que finaliza en la laguna. En el sitio se realizaron 10 sondeos en los lugares de mayor concentración de materiales, seis de ellos de 1 x $1 \mathrm{~m}$, y cuatro de 0,50 x 0,50 m. La mayoría de los sondeos fueron ampliados, efectuándose 9 anexos, todos de 0,50 x 0,50 m, con los que se alcanzó a un total de 9, $25 \mathrm{~m}^{2}$ de superficie excavada. Esto permitió reconocer una estratigrafía conformada por cuatro capas naturales, todas arqueológicamente fértiles: una capa superior limo-arenosa (capa 1), debajo de ésta, un paleosuelo poco desarrollado conformado por dos capas: una arcigilítica (capa 2) y una calcárea (capa 3), y finalmente, una capa arcillosa (capa 4) (Fig. 3). En la capa superior se hallaron débiles lentes de fogón, huesos con termoalteraciones y artefactos líticos. En la capa arcigilítica (capa 2), se hallaron abundantes materiales líticos, en su mayoría desechos de talla y escasos restos óseos, mientras que en la calcárea (capa 3) se rescataron dos manos de molino que afloraban del perfil de la cárcava mencionada. Por último, en la capa inferior se encontraron restos óseos asociados con carbones y solo seis lascas. Estos carbones fueron datados en $1.140 \pm 70$ años AP (LP-1731), lo que sitúa temporalmente a LF10 en el Holoceno tardío (Moreno \& Pérez op. cit.).

$\mathrm{Si}$ bien se pudieron diferenciar dos componentes, uno superior dentro de la capa 1 (limo-arenosa) y parte superior de la capa 2 (arcigilítica), y uno inferior dentro de la capa 4 (arcillosa), debido al muy escaso número de artefactos líticos de este último componente $(6$ lascas), solo se tomó en consideración para este análisis a los materiales del componente superior. Estos aparecen de forma dispersa, desde la superficie hasta aproximadamente los $40 \mathrm{~cm}$ de profundidad. Esta falta de estructuración sugiere, por oposición a otro sitio ya analizado del área (Delta Vulcana 1), la presencia de un palimpsesto (Peralta 2012). Con respecto a su ubicación temporal, la datación radiocarbónica mencionada, perteneciente al componente inferior, indica que estas ocupaciones tuvieron lugar en momentos posteriores a los 1.000 años AP.

El análisis faunístico de los materiales del componente inferior, señala la presencia de escasos restos de mamíferos grandes $(n=12)$, probablemente Lama sp., con un estado de conservación regular o malo. Con respecto al componente superior, los materiales óseos de la capa 1 poseen un mejor estado de conservación, y muchos de ellos, incluidos algunos de peces, presentan alteraciones térmicas. Se hallaron restos de Lama sp. $(\mathrm{n}=5)$, peces $(n=11)$-pejerrey patagónico (Odontesthes hatcheri) y perca (Percichtys sp.)-, pequeños roedores ( $\mathrm{n}=9$, posiblemente intrusivos), aves indeterminadas $(n=3)$, edentados $(n=13), y$ mamíferos indeterminados $(n=5)$. Además de estos restos óseos, se hallaron también varios fragmentos de cáscara de huevo de rheido (Moreno \& Pérez op. cit.).

\section{RESULTADOS DEL ANÁLISIS TECNO-MORFOLÓGICO}

El estudio tecno-morfológico fue desarrollado en base a los criterios tipológicos propuestos por Aschero (1975, 1983). Con respecto al conjunto de artefactos líticos tallados de LF10, conformado 


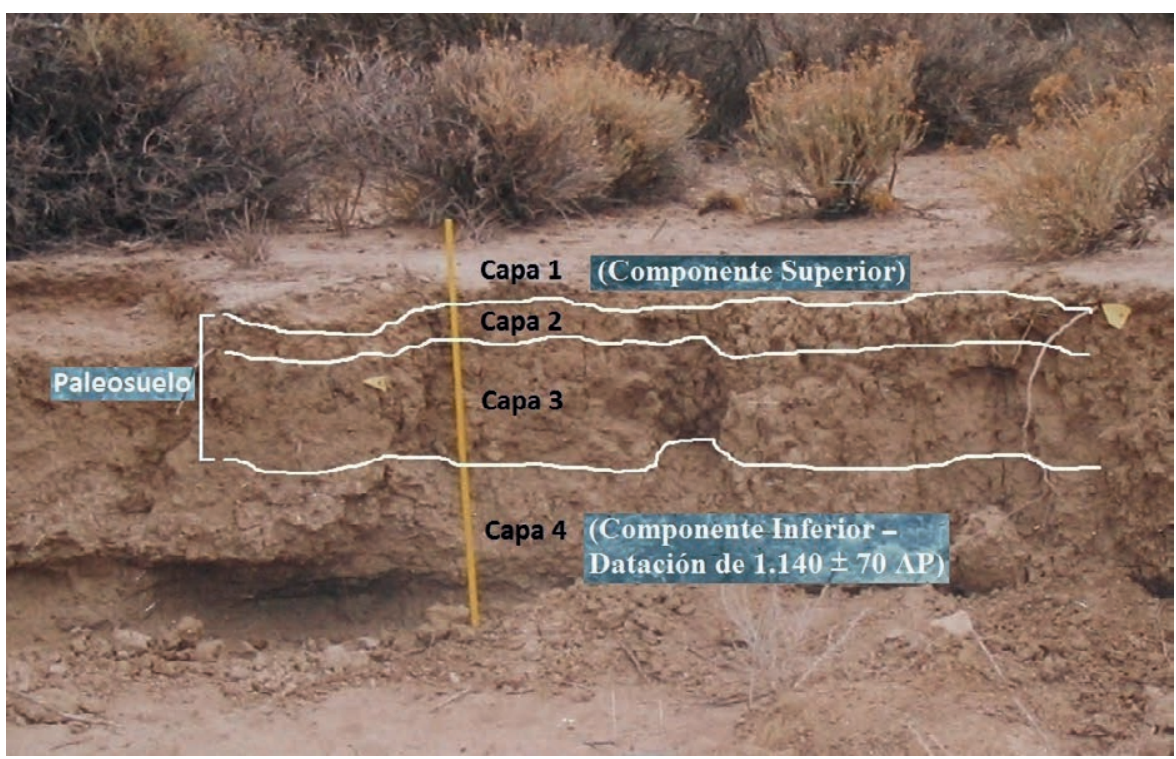

Fig. 3. Vista del perfil del sitio LF10, en la que se indican las capas y componentes identificados.

por 239 piezas, el análisis permitió conocer que en su mayoría corresponden a desechos de talla, siendo el porcentaje de los instrumentos el 7,5\% $(\mathrm{n}=18)$ y el de los núcleos el $1,7 \% \quad(\mathrm{n}=4)$. La clasificación tipológica de los instrumentos mostró un leve predominio de los artefactos formatizados, con el $61 \%(n=12)$ frente a aquellos con rastros de utilización, con el 39\% $(n=6)$. Considerando los grupos tipológicos, se destacan en primer lugar, por su frecuencia, los filos naturales con rastros complementarios, con el 33,3\% del total de instrumentos $(n=6)$, le siguen los raspadores, con el $22,2 \%(n=4)$, los bifaces, con el $16,6 \%$ $(\mathrm{n}=3)$, las puntas de proyectil, con un $11,1 \%$

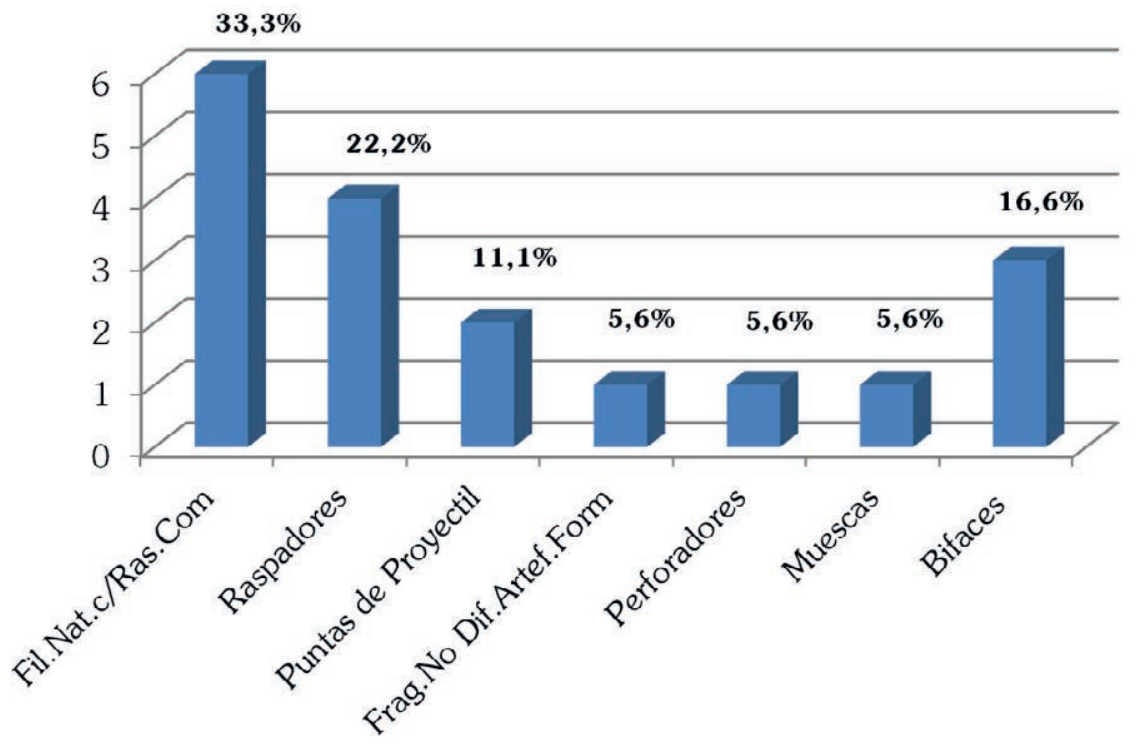

Fig. 4. Porcentajes de instrumentos líticos tallados, por grupo tipológico, de LF10.

(Referencias: Fil. Nat. c/ Rast. Com.: filos naturales con rastros complementarios; Frag. No Dif. Artef.

Form.: fragmentos no diferenciados de artefactos formatizados). 
$(n=2)$, y finalmente los grupos de las muescas, los perforadores y fragmentos no diferenciados de artefactos formatizados, cada uno con el 5,6\% $(n=1)$ (Fig. 4).

La muestra lítica total considerada en este trabajo la completan artefactos manufacturados por picado $y / o$ abrasión: dos fragmentos no diferenciados de artefactos de molienda de arenisca, dos manos de molino, ambas sobre rocas ígneas, y dos artefactos que habrían cumplido la función de pesas de red o línea, ambos recolectados en la superficie del sitio. Uno de ellos presenta una forma globular, irregular, lograda mediante picado, y surco perimetral (Fig. 5a); y el otro corresponde a un canto rodado plano, fracturado, y presenta tres muescas en sus bordes, manufacturadas por medio de abrasión y/o picado (Fig. 5b).

Con respecto a las puntas de proyectil halladas (ambas en la capa 1) son pedunculadas y están confeccionadas sobre variedades de sílice. Una de ellas conserva la parte proximal, presentando daños y una fractura oblicua en la zona del limbo que no permiten conocer la morfología del mismo, ni determinar la presencia de aletas u hombros; el pedúnculo es destacado, los bordes divergentes y la base cóncava (Fig.6a). La segunda, representa probablemente un caso de reciclaje, ya que en la zona del limbo muestra un estrechamiento muy marcado en forma de perforador. Presenta además, aletas salientes y un pedúnculo de bordes paralelos y una base cóncava (Fig. 6b). Piezas similares a esta fueron halladas una en superficie en la localidad Delta del Arroya Vulcana, en el sector noroeste del lago, y otra fue publicada por Molina para la costa norte de Santa Cruz (Molina 1976:85).

Con respecto a la elección de las formas base sobre las que fueron confeccionados los instrumentos tallados, entre las que pudieron ser identificadas se destacan las lascas internas, con el $16,7 \%(n=3)$, por sobre las lascas externas, con el $5,6 \%(n=1)$. Las lascas no diferenciadas representan el 33,3\% ( $n=6)$, y las formas base no diferenciada el $44,4 \%(n=8)$. El alto porcentaje de formas base no identificadas, responde principalmente al elevado índice de fragmentación

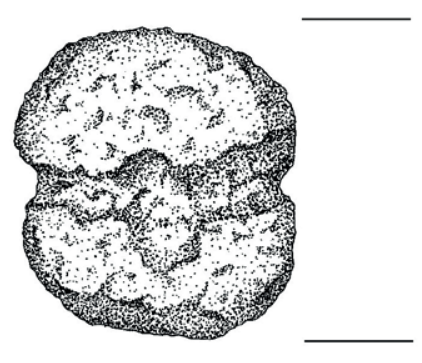

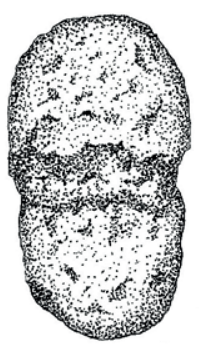

$\mathrm{b}$

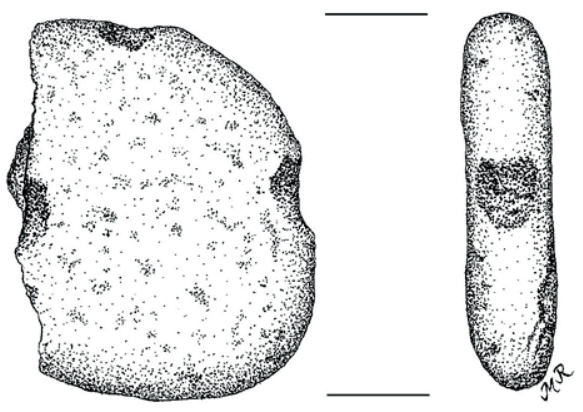

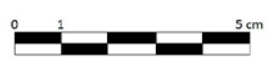

rig. ๖. Pesos líticos de LrIU. a

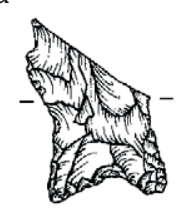

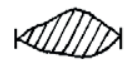

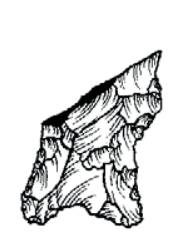

b

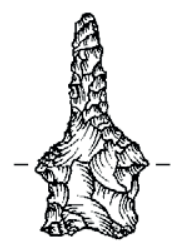

KLIIIIO

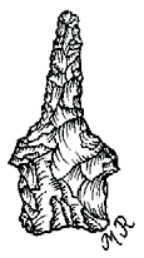

Fig. 6. Puntas de proyectil de LF10. 
de los instrumentos (66,7\% del total) y el alto grado de formatización de algunos de ellos.

En cuanto a los tipos de desechos, se destacan en primer lugar las lascas internas, con el $37,5 \%$ ( $n=63)$, a estas les siguen, con porcentajes similares, las lascas externas, con el $31,5 \%(n=53)$ y las de reducción o adelgazamiento bifacial, con el 29,2\% ( $n=49)$. Finalmente, en baja frecuencia, aparecen las lascas de reactivación de núcleo, con el $1,2 \%(n=2)$ y las lascas de reactivación de instrumentos, con el $0,6 \%(n=1)$.

Con respecto a los núcleos hallados $(n=4)$, tres de ellos corresponden a variedades de sílice: uno de tipo prismático parcial, bidireccional, con extracciones irregulares y de tamaño pequeño; otro de tipo prismático parcial, unidireccional, con extracciones irregulares, y de tamaño medianogrande; y el tercero, de tipo bifacial y tamaño pequeño. El restante, corresponde a una variedad de riolita, es de tipo bifacial y de tamaño medianogrande. Cabe destacar que todos ellos conservan restos de corteza, propias de clastos redondeados (rodados).

Por otro lado, los resultados del análisis de la distribución de los desechos enteros en intervalos de tamaño (Fig. 7) mostró un marcado predominio de los desechos pequeños. Tomando en conjunto los desechos de los tres primeros intervalos de tamaño (muy pequeños, pequeños y mediano- pequeños) alcanzan el $88,8 \%$ del total de desechos enteros.

Finalmente, los resultados del análisis de la distribución de la presencia de corteza en los desechos enteros según intervalos de tamaño (Fig. 8), indicaron una concentración de los restos de corteza en los intervalos de tamaños menores. Los desechos con corteza correspondientes a las categorías de tamaños pequeño y medianopequeños, alcanzan el 73,9\% del total.

\section{RESULTADOS DE LOS ANÁLISIS DE MATERIAS PRIMAS}

El análisis de materias primas se centró en las características macroscópicas de las mismas, tales como color, textura, inclusiones, etc., tanto del interior del material, como de la corteza cuando ésta estaba presente. Para su caracterización se utilizó una lupa binocular de la marca Zeiss, modelo Stemi 2000-C, con hasta 50x. A partir de este análisis se identificaron entre los artefactos tallados (desechos, instrumentos y núcleos), 125 variedades de rocas agrupadas en sílex, xilópalo, riolita y calcedonia. Cabe mencionar que, debido a la posible variación interna de las características macroscópicas que podrían presentar algunas de las rocas, este tipo de diferenciación de variedades, podría estar sobreestimando el número de las mismas $\mathrm{y}$, por

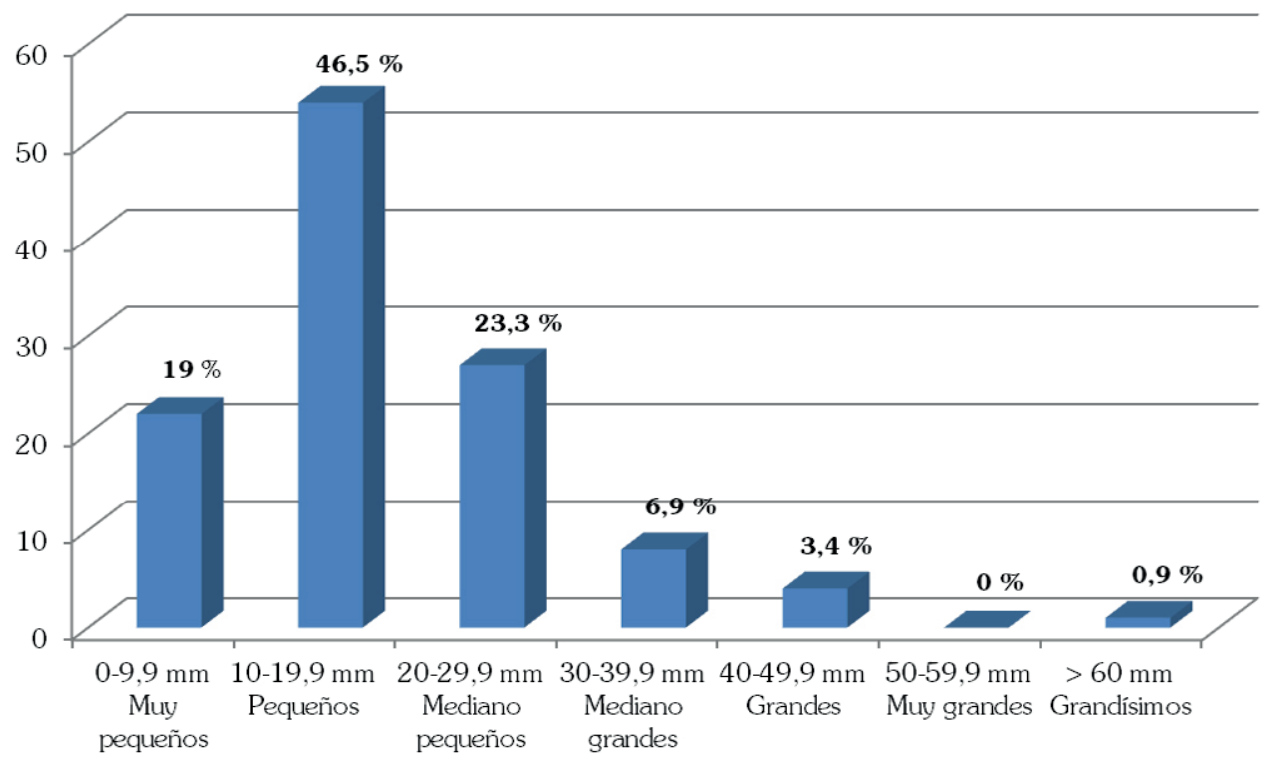

Fig. 7. Distribución de desechos enteros en intervalos de tamaño. 


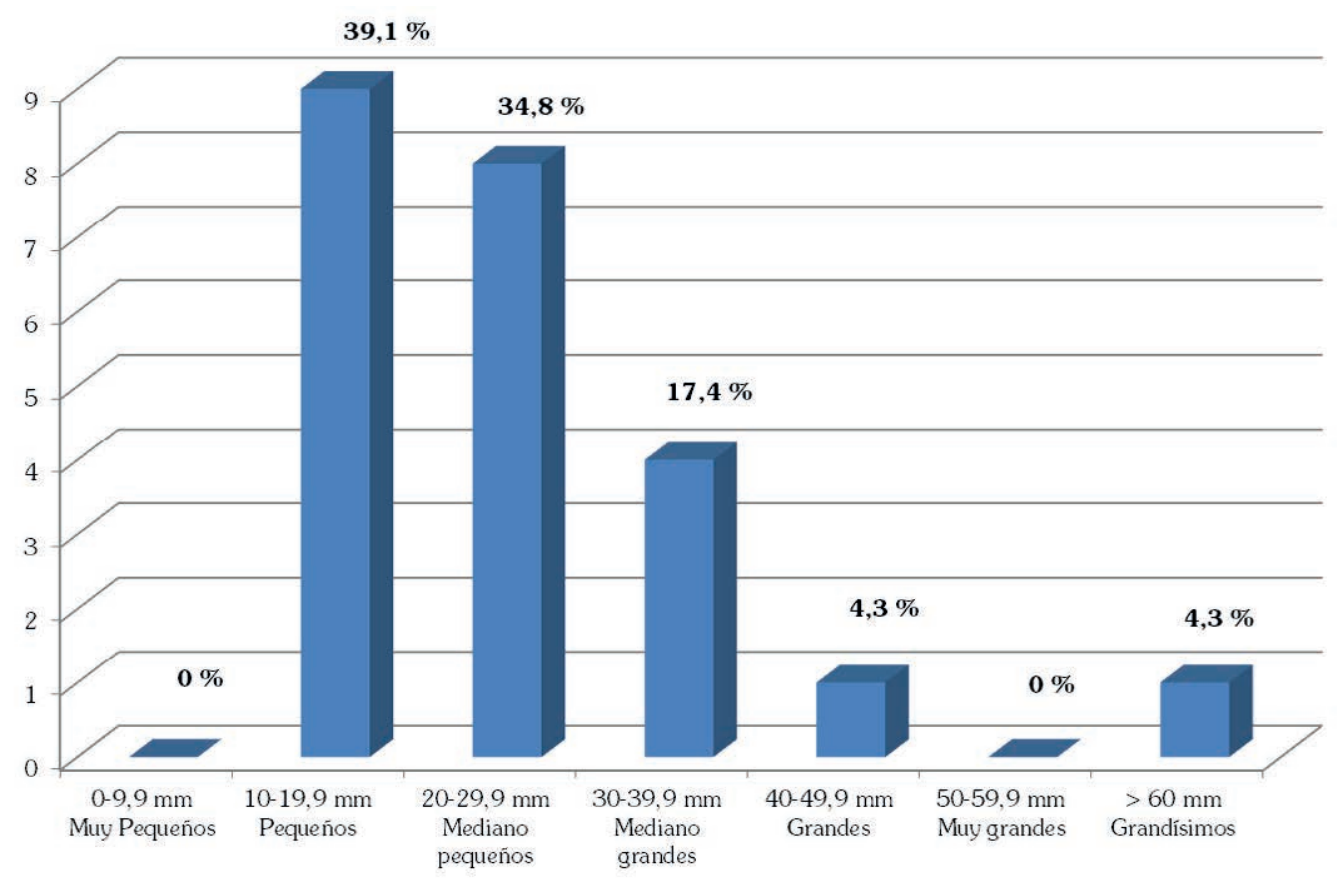

Fig. 8. Distribución de la presencia de corteza en desechos enteros según intervalos de tamaño.

ende, la cantidad de nódulos utilizados. Esta intravariación de las características macroscópicas de las materias primas, fue constatada en el análisis artefactual de otro sitio de la cuenca (Delta Vulcana 1), principalmente en los nódulos de xilópalos. En dicho análisis, los trabajos de remontaje resultaron de mucha ayuda ya que, en ocasiones, permitieron conocer que piezas de colores muy distintos pertenecían al mismo núcleo o nódulo.

Los resultados acerca de la distribución de las materias primas en los instrumentos indican que, en orden decreciente de su frecuencia estarian: el silex, con el 77,8\% ( $\mathrm{n}=14,14$ variedades), el xilópalo, con el $16,7 \%$ ( $n=3,3$ variedades), y finalmente la riolita, con el $5,5 \%(n=1)$. Como puede observarse, el sílex comprende la mayor parte de la muestra instrumental, mientras que el xilópalo y la riolita están escasamente representados (Fig. 9). Cabe mencionar que la cuenca se encuentra en un área rica en madera petrificada, con depósitos primarios y secundarios (Matheos et al. 2001), y si bien presenta grandes variaciones en cuanto a la calidad para la talla, ha sido explotada con intensidad, estando presente en la mayoría todos los sitios relevados.

Con respecto a la base de recursos líticos del área y a su disponibilidad, durante las prospecciones realizadas en el reconocimiento del sector y la búsqueda de sitios, se estuvo atento a la identificación de materias primas aptas para la talla, que podrían haber sido utilizadas. En este sentido, para evaluar dicha calidad, se realizó el testeo mediante percusión directa de numerosos clastos recolectados. Todo ello, nos ha servido para comenzar a familiarizarnos con la oferta de materia prima local más próxima y, en algunos casos, nos permitió además proponer, a modo de hipótesis a avaluar con futuros trabajos, posibles correspondencias a nivel macroscópico de materias primas locales (de la cuenca lacustre) con algunas de las presentes en los artefactos del sitio. Estas se presentan en forma de rodados en extensos mantos en zonas elevadas de la cuenca. El deposito con estas características más próximo al sitio, es un cordón litoral distante unos 100 m, hacia el Este.

Finalmente, con respecto a la procedencia de las materias primas de los artefactos formatizados mediante picado y/o pulido, tanto las areniscas de los dos fragmentos no diferenciados de artefactos de molienda, como las rocas ígneas de las dos manos de molino y de una de las dos pesas de red o línea, se encuentran presentes en varios 


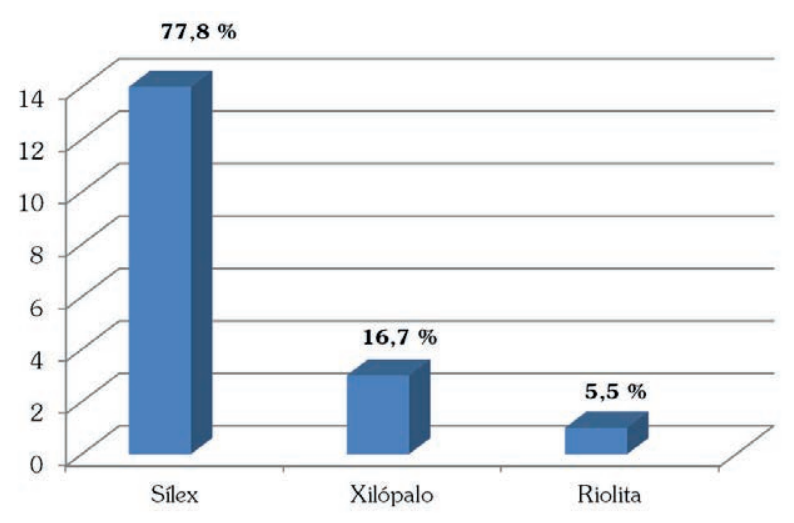

Fig. 9. Porcentajes de materias primas líticas en instrumentos tallados de LF10.

afloramientos y fuentes secundarias de la cuenca. El afloramiento de arenisca más cercano al sitio, se encuentra a unos $2000 \mathrm{~m}$ hacia el Este, y pertenece a las formaciones Bajo Barrial y Castillo; en tanto que las ígneas podrían corresponder a Basaltos Buen Pasto, cuyo afloramiento más cercano se encuentra a unos $3000 \mathrm{~m}$ hacia el Noreste.

\section{CONSIDERACIONES FINALES}

Teniendo en cuenta la variedad relativamente grande de los tipos de instrumentos, con la presencia entre ellos de artefactos de molienda, podríamos caracterizar al sitio LF10 como un sitio de actividades múltiples. Al respecto, el estudio tipológico muestra evidencia indirecta de procesamiento y consumo de animales y vegetales, procesamiento de cueros ${ }^{1}$ $y$ actividades de talla. Por otro lado, la presencia de puntas de proyectil y pesas líticas sugieren el desarrollo de actividades de caza y pesca. Con respecto a estos últimos artefactos, uno de ellos, el confeccionado sobre un guijarro plano (Fig. 5b), por su morfología, dimensiones y presencia de muescas, se asemeja a las pesas de red o de línea descriptas etnográficamente para Tierra del Fuego (Orquera \& Piana 1999:230) como también a los ejemplares arqueológicos publicados para la costa del Golfo San Matías (Scartasini \& Cardillo 2009), y para el norte de Tierra del Fuego (Torres 2007). La actividad de pesca se infiere además, por la presencia en el sitio de huesos de peces, algunos

1 Si bien serán los estudios funcionales de base microscópica los que permitan confirmar eventualmente la presencia del procesamiento de cueros, por lo general, estos estudios en de ellos termoalterados (Moreno \& Pérez op. cit.).

Sin embargo, teniendo en cuenta el reducido tamaño del conjunto lítico y, dentro de éste, el de instrumentos, el sitio habría sido ocupado con baja intensidad. Por otra parte, los resultados dan cuenta de un marcado predominio de desechos de talla, y la presencia de todas las instancias de reducción de las materias primas. Las mismas provendrían, probablemente, de sectores cercanos, y habrían sido introducidas al sitio en forma de clastos redondeados, de tamaños predominantemente pequeños, como estarían indicando los núcleos con restos de corteza y el alto porcentaje de lascas de descortezamiento pequeñas. Estos clastos pudieron haber sido recolectados en los mantos de rodados que cubren amplias superficies de las zonas más elevadas de la localidad y áreas vecinas. En este sentido cabría pensar en una estrategia de aprovisionamiento incidental (embedded) sensu Binford (1979), llevado a cabo, posiblemente, durante los traslados hacia sectores de caza o recolección de otros recursos. Esta hipótesis se pondrá a prueba a través de nuevos y más detallados análisis de las materias primas (geoquímicos y de cortes delgados) y estudios de potenciales fuentes de aprovisionamiento. En este sentido, se hace necesaria la continuación de los trabajos de campo, en la búsqueda de otras fuentes potenciales, con la toma de muestras para su comparación con lo hallado en este y otros sitios de la cuenca. Por otro parte, se plantea la necesidad de realizar análisis funcionales de base microscópica, a fin de aproximarnos de una manera más precisa a las tareas que habrían desempeñado los artefactos.

Los resultados obtenidos en este trabajo indican una semejanza en las tendencias tecnológicas entre LF10 y Delta Vulcana 1, el otro sitio de la cuenca cuyos materiales han sido analizados. Este último sitio, perteneciente también al Holoceno tardío (ca 1.400 AP) si bien presenta, a diferencia de LF10, una alta resolución e integridad, comparte con él varias características. Entre ellas están la alta diversidad artefactual, con la presencia también de instrumentos de molienda, la utilización de rodados de tamaño pequeño, la densidad artefactual, la proporción entre los

Patagonia tienden a relacionan al tipo morfológico "raspador" con el procesamiento de cuero. 
desechos de talla e instrumentos, el predominio de los artefactos formatizados sobre los artefactos con rastros de utilización, el marcado predominio de los desechos de tamaños pequeños, etc. Por otra parte, en cuanto al registro arqueofaunístico se constató también la presencia de restos de guanaco y peces (percas) (Moreno \& Pérez op. cit.). Todo esto nos llevaría a suponer que ambos sitios habrian corresponderían a ocupaciones residenciales. Así mismo, de acuerdo a las observaciones preliminares sobre la oferta de materias primas locales de ambos sitios, permitirian proponer a modo de hipótesis estrategias de aprovisionamiento similares.

Esperamos que el avance de las investigaciones, principalmente con la extensión de las tareas de excavación sobre nuevos sitios de la localidad y el desarrollo de estudios referidos a la base de recursos, permita ampliar nuestro conocimiento de las estrategias en el manejo de recursos líticos de estos grupos.

\section{AGRADECIMIENTOS}

Al Consejo Nacional de Investigaciones Científicas y Tecnológicas y a la Universidad Nacional de la Patagonia San Juan Bosco, instituciones que financiaron estas investigaciones. Al Dr. Eduardo Moreno por sus valiosas sugerencias. Y a los evaluadores anónimos y miembros de la Comisión Editora, quienes contribuyeron a mejorar este trabajo.

\section{BIBLIOGRAFÍA}

Aschero, C. A. (1975). Ensayo para una clasificación morfológica de artefactos líticos aplicada a estudios tipológicos comparativos. Informe presentado al CONICET, Argentina.

Aschero, C. A. (1983). Ensayo para una clasificación morfológica de artefactos líticos. Apéndice A y B. Cátedra de Ergología y Tecnología. Facultad de Filosofía y Letras, Universidad de Buenos Aires.

Binford, L. R. (1979). Organization and formation processes: looking atcurated technologies. Journal of Anthropological Research, 35, 255-273.

Binford, L. R. (1980). Willow Smoke and Dog's Tails: Huntergatherer Settlement Systems and Archaeological Site For-mation. American Antiquity, 45, 4-20.

Bórmida, M. (1956). Arpones de hueso de la Patagonia
Meridional. Runa, 7, 242-244.

Castro, A., Pérez de Micou, C., Burry, L.S. \& Trivi de Mandri, M. (2007). Paleoambiente y etnohistoria en el lago Colhué Huapi. En Actas del XVI Congreso nacional de Arqueología Argentina, San Salvador de Jujuy.

Flannery, K. V. (1969). Origins and ecological effects of early domestication in Iran and the Near East. En Ucko \& G.W. Dimbleby (Eds), The domestication and exploitation of plant and animals (pp. 73-102). Londres: Editorial Gerald Duckwoth \& Co.

González, A. R. (1953). Las boleadoras, sus áreas de dispersión y tipos. Revista del Museo de la Ciudad de Eva Perón, Sección Antropología, 4, 133-292.

Matheos, S., Brea, M.; Ganuza, D. \& Zamuner A. (2001). Sedimentología y paleoecología del terciario inferior en el sur de la Provincia del Chubut. República Argentina. Revista de la Asociación Argentina de Sedimentología, 8, 93-104.

Molina, M. J. (1967-70). Arpones monodentados de la Patagonia Meridional. Acta Prehistoria, VIII/X (1), 173-179.

Molina, M. J. (1976). Patagónica. Prehistoria, Tradiciones y Mitologías. Roma: Editorial LAS.

Moreno, E., Videla, B., Pérez Ruiz, H., Asencio, L. \& Leonforti V. (2007). Búsqueda de indicadores de diversificación económica prehistórica en la cuenca del lago Musters (Chubut, Argentina), primeros resultados. En F. Morello, M. Martinic, A. Prieto y G. Bahamonde (Eds.), Arqueología de Fuego Patagonia. Levantando piedras, desenterrando huesos...y develando arcanos (pp.23-32). Punta Arenas: Ediciones CEQUA.

Moreno, E. \& Pérez Ruiz, H. (2010). Evidencias de utilización prehispánica de recursos fluviales en la cuenca del lago Musters (Chubut, Argentina). En Actas del XVII Congreso Nacional de Arqueología Argentina (pp. 345- 350), Mendoza.

Nelson, M. C. (1991). The Study of Technological Organization. En M. Schiffer (Comp.), Archaeological Method and Theory, vol 3 (pp. 57-100). Tucson: University of Arizona Press.

Orquera, L. \& Piana, E. (1999). La vida material y social de los Yámanas. Buenos Aires: Editorial EUDEBA.

Outes, F. (1905). La edad de la piedra en Patagonia. Anales del Museo Nacional de Buenos Aires, 12(5), 203-575.

Peralta González, S. (2012). Movilidad y patrón de asentamiento de cazadores-recolectores durante el Holoceno Tardio en Patagonia Central: Análisis intrasitio del caso de Delta del Arroyo Vulcana 1 (lago Musters, Chubut). Tesis de Licenciatura en 
Historia, Universidad de la Patagonia San Juan Bosco, Comodoro Rivadavia.

Pérez de Micou, C., Castro, A., Funes, M. L., Burry, S. \& Trivi, M. (2009). Prospecciones en el Río Chico, provincia de Chubut. En M. Salemme, F. Santiago, M. Álvarez, E. Piana, M. Vázquez \& M. Mansur (Eds.), Arqueología de la Patagonia: una mirada desde el último confín, Vol 2 (pp.1149-1158). Ushuaia: Editorial Utopias.

Quirós, R. (1988). Relationships between air temperature, depth, nutrients and chlorophyll in 103 argentinian lakes. Verhandlunge Internationale Vereinigung für Theoretische und Angewandte Limnolgie, 23, 647658.

Reyes, M., Peralta González, S. \& López Ferrer A. L. (2013). Análisis preliminar de los materiales líticos del sitio Delta Vulcana 1 (lago Musters, Chubut). En A. F. Zangrando, R. Barberena, A. Gil, G. Neme, M. Giardina, L. Luna, C. Otaola, S. Paulides, L. Salgán \& A. Tivoli (Eds.), Tendencias teórico-metodológicas y casos de estudio en la arqueología de la Patagonia (pp. 219226). Mendoza: Edición del Museo de Historia Natural de San Rafael.
Scartascini, F. L. \& Cardillo, M. (2009). Explorando la variabilidad métrica y morfológica de las "Pesas líticas" recuperadas en el sector Norte de la costa del golfo San Matías. En O. Palacios, C. Vázquez, T. Palacios \& E. Cabanillas (Eds.), Arqueometría latinoamericana: Segundo Congreso Argentino y Primero Latinoamericano (pp. 162-168). Buenos Aires: Comisión Nacional de Energía Atómica.

Smith, E. A. (1985). Anthropological applications Of optimal Forraging Tehory: a critical review. Current Anthroplogy, 24(5), 625-651.

Stiner, M., Munro, N. \& Todd, S. (2000). The tortoise and the hare, small-game use, the broad- spectrum revolution and the Paleolithic demography. Current anthropology, 41(1), 39-73.

Torres, J. (2007). ¿Redes o líneas de pesca?: el problema de la asignación morfofuncional de los pesos líticos y sus implicancias en las tácticas de pesca de los grupos del extremo austral. Magallania, 35(1), 53-70.

Vignati, A. M. (1950). Estudios antropológicos en la zona militar de Comodoro Rivadavia. Anales del museo de La Plata. Antropología, Sección Antropología, 1, 7-18. 